\title{
Observations with the electron microscope on the absorption of India ink from the peritoneal cavity of the rabbit
}

By

\author{
Masahiko Kotani, Masao Rai and Shiro Nakao \\ From the Second Division of Department of Anatomy, \\ Faculty of Medicine, Kyoto University, Kyoto \\ (Director: Prof. I. Hori i) \\ Seven Plates (22 Figures)
}

\section{Introduction}

It has been well known that inert foreign materials, introduced into the peritoneal cavity, are easily and rapidly absorbed by the lymphatic vessel on the surface of the diaphragm as cited briefly in Lymphatics, Lymph and Lymphoid Tissue by Yoffey and Courtice (1956) or in Physiologie und Pathologie des Lymphkreislaufes by $\mathrm{Ruszuyák}, \mathrm{Föld}$ i and $\mathrm{Szabó}$ (1957). The pinocytotic activity of the peritoneal cells of the rat's diaphragm, observed with the electron microscope by Odor (1956), explains one of the most important mechanisms of absorption from the peritoneal cavity. However, many problems as yet remain. In order to study the absorption from the peritoneal cavity into the lymphatics, the site of most intensive absorption where the lymphatic capillaries distribute beneath the peritoneum must be used for the observations. In the rabbit, the lymphatic absorption from the peritoneal cavity takes place almost wholly at the tendon parts of the diaphragm. The lymphatic capillaries run parallel with one another between the parallel lying tendon bundles, although they are abundantly connected by the oblique or transverse rnnning, anastomosing lymphatics. The findings using the light microscope by $\mathrm{kih}$ a r (1956) and his scholars have shown that the peritoneal cells covering the area where the lymphatic capillaries distribute are remarkably smaller in size than the common cells. Moreover, the collagenous bundles in the submesothelial layer are loosely arranged beneath the smaller peritoneal cells, although they are compact in the area where the lymphatics do not distribute, and the intraper- 
itoneal injected carbon particles stick particular richly to the intercellular spaces between the smaller peritoneal, cells and to the meshes between the loosely arranged collagenous bundles.

\section{Materials and Methods}

The rabbits, 2.2 to $2.5 \mathrm{~kg}$ in weight, were killed by air embolus 15, 30, 60 minutes after intraperitoneal injection of 20 c. c. of India ink. As soon as the animals were sacrified, the tendon parts of the diaphragm were quickly taken out, immersed in the chilled 1 per cent osmium tetroxide in phosphate buffer ( $\mathrm{pH}: 7.4)$. After fixation for 2 hours, the materials were washed in distilled water. The small tissue pieces between the parallel running tendon bundles, heavily stained with India ink, were carefully cut off, dehydrated through increasing concentrations of ethanol alcohol and embedded in a mixture of 40 parts methyl methacrylate and 60 parts n-butyl methacrylate. Thin sections, cut with a glass knife on a Nippon Microtome Laboratory RU-5 M microtome, were stained with lead hydroxide and examined with Hitachi HU-10 or HS-6 electron microscope. It was very difficult to make thin sections of the tissue, heavily loaded with the carbon coagulations.

\section{Observations}

The carbon particles, introduced into the peritoneal cavity, were abundantly found not only in the lumen of the lymphatic capillaries, but also in the large spaces between the submesothelial collagenous bundles in all specimens from 15 to 60 minutes after injection (Figs. 2 to 21). This fact suggests that the submesothelial tissue spaces play an important rôle in carrying carbon particles from the peritoneal cavity to the lymphatic capillary. These spaces were separated from the peritoneal cavity by the peritoneal cell lining and from the lumen of the lymphatic capillary by the lining of the lymphatic endothelial cells. Frequently, thin cytoplasmic processes of the lymphatic endothelial cells extended on the surface of the collagenous bundles to come in direct contact with the peritoneal cells, and the submesothelial tissue space was surrounded, as a whole, by the cell elements (Figs. 17 and 18). The submesothelial space was occasionally divided into a few small spaces by thin transversely or obliquely running cytoplasm of the lymphatic endo- 
thelial cells, whearas small spaces communicated through the interruption of the cytoplasm with each other (Fig. 21). The size of the carbon particles in the submesothelial space or in the lumen of the lymphatic capillary was the same with those in the peritoneal cavity. The average diameter was measured between $40 \mathrm{~m} \mu$ and $55 \mathrm{~m} \mu$.

The carbon particles, injected into the peritoneal cavity, must pass the peritoneal cell layer to enter the submesothelial tissue space. The observations with the lectron microscope confirmed the findings with the light microscope that the peritoneal cells lining the intensive absorption parts of the diaphragm were in general smaller in size, but higher in statue than those covering the rest parts. The peritoneal cells showed a large number of the microvilli-like processes projecting into the peritoneal cavity. They were also rich in small vesicles in the cytoplasm. The basement membrane could not be observed beneath the peritoneal cells in the intensive absorption parts of the diaphragm. The peritoneal cells were often attached heavily on their surfaces, facing the peritoneal cavity, by the very small carbon particles (Figs. 4, 6, 17-20). On the contrary, the peritoneal cell surfaces, facing the submesothelial tissue space, were rarely attached by the small particles. The common sized carbon particles were frequently located at the base of the microvilli-like processes of the cytoplasm (Figs. 14, 15, 22). Occasionally, the carbon particles seemed to stick to the vesicles or small vacuoles opening to the peritoneal cavity (Figs. 1, 10, 21). The carbon particles could be found in the peritoneal cell layer either in the intercellular space or in the cytoplasm. It must be noticed that the carbon particles, ingested into the peritoneal cell, were few or absent at 15 minutes after injection, although the submesothelial tissue spaces and the lumen of the lymphatic capillaries were already abundant in the carbon particles. At 15 to 30 minutes after injection, the carbon particles situated mainly in the intercellular spaces between the peritoneal cells. The uptaken carbon particles in the cytoplasm of the peritoneal cells fairly increased in number at 30 minutes and remarkably at 60 minutes after injection.

The intercellular spaces were often dilated like a canal at the junctions of the thick cytoplasms of the peritoneal cells (Figs. 1, 11, 14-16). Moreover, the local, large dilations of the intercellular spaces were frequently observed (Figs. 1, 8, 14). At the junctions 
of the thin cytoplasms of the peritoneal cells, two neighbouring cells were often widely separated with each other, at a maximum of about $3 \mu$ width (Figs. 2, 3, 7, 18, 19). Through this wide space between two separated peritoneal cells, the peritoneal cavity opens to the large submesothelial space, and the lymphatic endothelial cell layer seemed to be directly exposed to the peritoneal cavity. The carbon particles were often continuously found from the peritoneal cavity through a canal-like intercellular space or a space between two widely separated peritoneal cells to the submesothelial tissue space. The carbon particles in the peritoneal cells were situated in the vacuoles, limited by the thin membrane (Figs. 10, 14, 16-18, 20). The vacuoles containing the carbon particles were various in size and shape, and the largest one reached $1 \mu$ or more at its long axis. The size of each carbon particles in the vacuoles were similar to those in the extracellular spaces. The vacuoles occasionally opened to the submesothelial space to discharge the carbon granules (Figs. 17 and 21). Rarely, occurred the deep invaginations of the cell membrane, which seemed to be in close relation to the uptake or excretion of the particles (Fig. 11). The carbon particles, moreover, appeared in the oval opaque bodies in the cytoplasm of the peritoneal cells (Figs. 1, 18, 20-22). The average diameter of the opaque bodies was about 0.5 to $0.6 \mu$ at their major axis. The occurrence of the opaque bodies was not frequent throughout these experiments. However, it must be noted that the opaque bodies showed a tendency of increasing in number with the time after injection. The carbon particles in the opaque bodies were in general not so large in number as in the vacuoles. Moreover, it was interesting to note that very small particles appeared in the opaque matrix with a few, rather large particles. An oval structure, limited by a single highly dense membrane, was often contained in the opaque body with the carbon particles (Figs. 20 to 22). It was around 0.2 to $0.3 \mu$ in diameter.

The carbon particles, carred from the peritoneal cavity into the submesothelial space, must penetrate the wall of the lymphatic capillary to enter its lumen. The wall of the lymphatic capillary provided for neither the basement membrane nor the perivascular cell. The cytoplasm of the lymphatic endothelial cells was abundant in the small vesicles. The carbon particles frequently seemed to stick to the vesicles or small vacuoles opening to the submesothelial tissue space (Figs. 2, 7, 20). Occasionally, the carbon particles were 
found at the base of the small, microvilli-like cytoplasmic processes, projecting into the submesothelial space (Fig. 20). In the lymphatic endothelial layer, the carbon particles could be found in the intercellular spaces between the neighbouring lymphatic endothelial cells and in the vacuoles in the cytoplasm of the lymphatic endothelial cells, as well as in the peritoneal layer. The intercellular spaces were of ten dilated, like a canal, to connect the submesothelial space to the lumen of the lymphatic capillary (Figs. 6, 9, 11, 17). The local dilation of the intercellular space was also observed (Figs. 1, $8,10,18)$. The phagocytotic vacuoles in the cytoplasm of the lymphatic endothelial cells were varied in size and shape (Figs. 7, 8, 18-20). Frequently, they reached a great diameter. Apparently, the vacuoles, including the carbon particles, opened directly or through the tubular invagination of cell membrane into the lumen of the lymphatic capillary (Figs. 18 and 19). One of the differences between the lymphatic endothelium and the peritoneal mesothelium was that the phagocytosis by the lomphatic endothelial cells could be seen more frequently in the specimens after early injection than by the peritoneal cells. The opaque body did not appeared in the cytoplasm of the lymphatic endothelial cells. Rarely, was the surface of the lymphatic endothelial cells, facing the submesothelial space, slightly attached by the extremely small carbon particles as on the free surface of the peritoneal cells (Fig. 19).

In the sections through the area where an anastomosing lymphatic vessel between the parallel running lymphatics ran far from the general level of the peritoneum, a deep invagination of the peritoneal cell lining occurred in order to approach the peritoneal cavity to the lymphatics (Figs. 4 and 5). On the other hand, a cytoplasmic process of the lymphatic endothelial cell extended to the peritoneal cells. In a portion of the section, the carbon particles entered through a opening in the peritoneal cell layer of the invagination into the submesothelial tissue space, and attached to the process of the lymphatic endothelial cell (Fig. 5). In another portion of the same section, the carbon particles were carried for a long way through the tissue spaces in deeper layer to the outside of the wall of the lymphatics, and then entered through a tubular dilated intercellular space between the lymphatic endothelial cells into the lumen of the lymphatic vessel (Fig. 6). 


\section{Discussion}

The large, submesothelial spaces between the collagenous bundles, playing an important rôle in carrying the carbon particles, must correspond to the meshes of the macula cribriformis, named by K ih a r a (1956). The intercellular space between the lymphatic endothelial cells has been electron microscopically indicated as a pathway of fat absorption into the lacteal lymphatics by $\mathrm{P}$ a $\mathrm{l}$ a $\mathrm{y}$ and $\mathrm{K}$ a r l in (1959). The present work has shown that the intercellular space between the peritoneal mesothelial cells, as well as the intercellular space between the lymphatic endothelial cells, is a passage for the carbon particles. The carbon particles are taken up into the vesicles, opening to an extracellular space by means of the micropinocytotic activity or into the small vacuoles by means of the enveloping of the microvilli-like processes. The large vacuoles, including the carbon particles in the cytoplasm, might be caused by a fusion of the vesicles or small vacuoles. The vacuoles open to the opposite side to discharge the carbon particles into another extracellular space. The transference of abundant carbon particles may take place by means of the deep invagination of the cell membrane, connected to the large vacuole. The appearance of the foreign materials in a body having a relatively dense matrix has been shown by Od or (1956) in the uptake of the mercuric sulfide particles. The carbon particles also appeared in the dense bodies in the cytoplasm of the peritoneal cells. These bodies apparently look like one of the profiles of the segresome, reported by $\mathrm{T} \mathrm{a} \mathrm{nak}$ a (1960). The extremely small particles, attached to the free surface of the peritoneal cells, may be in close relation to the segregation.

Very recently, we received a report by $\mathrm{Y}$ a m a gishi (1961) after we had finished making the present paper. According to his findings, most of the China ink particles, injected into the peritoneal cavity of the dog, were absorbed through the interspaces between the lymphatic endothelial cells into the lymph vessels of the diaphragm, and partly phagocytized by the vesicles in the cytoplasm of the endothelial cells. Moreover, the lymph vessels in the diaphragm of the dog provided for neither the basement membrane nor the pericytes. 


\section{Summary}

1) The India ink particles, introduced into the peritoneal cavity, entered the lymphatic capillaries in the tendon parts of the diaphragm through the submesothelial tissue spaces between the collagenous bundles. Frequently, thin cytoplasmic processes of the lymphatic endothelial cells extended on the surface of the submesothelial collagenous bundles to come in direct contact with the peritoneal cells.

2) The India ink particles were carried from the peritoneal cavity through a dilated, canal-like intercellular space between two neighbouring peritoneal cells, or through a opening between two widely separated peritoneal cells into the submesothelial tissue space. The India ink particles were also transferred through the cytoplasm of the peritoneal cells. In the cytoplasm, the carbon granules was situated in the vacuoles, varying in size and shape, and in the opaque bodies. The vacuoles including the carbon granules were greatly increased in number at 60 minutes after injection, although the intercellular spaces were the main pathway at 15 to 30 minutes after injection.

3) From the submesothelial tissue space the carbon particles were transported through the intercellular spaces between the lymphatic endothelial cells, or through the vacuoles in the cytoplasm of the lymphatic endothelial cells into the lumen of the lymphatic capillaries.

\section{Acknowledgements}

We are grateful to Professor I. Hor i i for his guidance and interests throughout this work. We are also indebted to Professor H. $\mathrm{N}$ is h i m u ra for his support and encouragement, and to Emeritus Professor T. K i h a r a for his criticisms during the progress of this work. We wish to express sincere thanks to $\mathrm{H}$. T a n a k a of the Virus Institute for his helpful advice and suggestion. Our thanks are also due to Mr. S. Uchida and Mr. M. Fujioka of the Electron Microscope Center for their kind technical aids. 


\section{Bibliography}

1) Felix, M.D. and Dalton, A. J.: A comparison of mesothelial cells and macrophages in mice after intraperitoneal inoculation of melanin granules. J. Biophysic. and Biochem. Cytol., 1956, 2, No. 4, suppl., 109.

2) K ihara, T.: Das extravaskuläre Saftbahnsystem. Okajimas Fol. anat. jap., 1956, 28, 601 .

3) Odor, D.L.: Uptake and transfer of particulate matter from the peritoneal cavity of the rat. J. Biophysic. and Biochem. Cytol., 1956, 2, No. 4, suppl., 105.

4) Rusznyák, I., Földi, M. and Szabó, G.: Physiologie und Pathologie des Lymphkreislaufes. Fischer, 1957.

5) T a n a k a, H.: Electronenoptische Beobachtungen über die vitalen Karminspeicherung (Japanese). Acta Haem. Jap., 1960, 23, No. 2, 447.

6) Ya magishi, T.: Fine structure of lymph vessels in the abdominal wall of the diaphragm of dogs, and their attitude following absorption of China ink. Nagoya Med. J., 1961, 7, No. 1, 1.

7) Yof fey, J.M., and Courtice, F.C.: Lymphatics, Lymph and Lymphoid Tissue. Arnold, 1956.

\section{Explanation of Figures}

Plate 1

Figs. 1 to 3 are from the animals killed 15 minutes after intraperitoneal injection of India ink.

Fig. 1. The carbon particles lie at the entrance of a deep and wide dilation of the intercellular space between the two neighbouring peritoneal cells (m). A few carbon particles apparently stick to the free surface of the peritoneal cells (arrow). A opaque body (o) in the cytoplasm of the peritoneal cell includes the carbon particles. Beyond the submesothelial connective tissue (c), a local dilation of the intercellular space between the two neighbouring endothelial cells (e) of a lymphatic capillary $(\mathrm{L})$ can be seen. The peritoneal cavity is indicated at $\mathrm{P}$. $\times 24,000$.

Figs. 2 and 3. The carbon particles can be seen passing from the paritoneal cavity (P) through a wide opening in the peritoneal cell (m) layer to a large, submesothelial space (s) between the collagenous bundles (c). The submesothelial tissue space is separated from the lumen of a lymphatic capillary (L) containing abundant carbon particles by its endothelial cell (e) layer. The carbon particles stick to a few vesicles or small vacuoles in the thin cytoplasm of the lymphatic endothelial cell. Fig. $2, \times 23,000$ Fig. $3, \times 24,500$.

Plate 2

Figs. 4 to 6 are from an animal killed 15 minutes after intraperitoneal injection of India ink. These three figures are succeeding. The sections were made through a place where an anastomosing lymphatic vessel between the parallel running lymphatics lie far from the general level of the peritoneum.

Fig. 4. The free surface of the peritoneal cells $(m)$ is attached by the very small carbon particles. A deep invagination of the peritoneal cell lining occurs in the left one third of this figure. Abundant carbon particles can be seen at the en- 
trance and in the lumen of the invagination. Adjacent to the right upper corner, a large number of carbon particles lie continuously from a intercellular space between the peritoneal cells to a submesothelial tissue space. The processes of an endothelial cell (e) of the lymphatic vessel in the deep layer extend and contact with the peritoneal cells. Two large spaces between the submesothelial connective tissue (c) and the lymphatic endothelial cell (e) is almost completely surrounded by the processes of a cell which is uncertain, but may be a endothelial cell of the lymphatic vessel in the superficial layer. Three groups of abundant carbon particles can be seen in the spaces surrounded by the uncertain cell, the peritoneal cell and the lymphatic endothelial cell. The peritoneal cavity is indicated at $P . \times 13,000$.

Fig. 5. The peritoneal cavity $(\mathrm{P})$ communicates with a submesothelial space through a wide opening (arrow) in the invaginated peritoneum which is closed in figure 4. Abundant carbon particles in another submesothelial space reached the surface of a lymphatic endothelial cell (e) and the submesothelial connective tissue (c). The peritoneal cells are shown at $\mathrm{m} . \times 14,500$.

Fig. 6. The carbon particles can be seen in the peritoneal cavity $(P)$ and in the submesothelial spaces, covered at its major part by the processes of a uncertain cell. There are two groups of carbon particles in the connective tissue (c) spaces, in which the deeper one lies outside of the lymphatic endothelial cells (e). An interrupted tubular space (arrow) between the lymphatic endothelial cells (e) connects the deeper tissue space to the lumen of the lymphatic vessel (L). $\times 8,000$.

Plate 3

Figs. 7 to 9 are from the animals killed 15 minutes after intraperitoneal injection of India ink and Fig. 10 is from an animal killed 30 minutes after injection.

Fig. 7. The carbon particles can be seen passing from the peritoneal cavity (P) through a wide space between the peritoneal cells $(\mathrm{m})$ to a large submesothelial tissue space (s). Another intercellular space between the peritoneal cells is tightly closed. The submesothelial space is separated from the lumen of a lymphatic capillary (L) by its endothelial cell (e) lining. The carbon particles apparently stick to the surface of the lymphatic endothelial cell. Near the left lower corner, a vacuole in the cytoplasm of the lymphatic endothelial cell contains a few carbon particles. $\times 21,000$.

Fig. 8. The peritoneal cells (m) and the lymphatic endothelial cells (e) are in direct contact with each other. The carbon particles can be seen not only in the two small vacuoles (arrow) in the cytoplasm of the lymphatic endothelial cell, but also in the local dilations of the intercellular spaces between the peritoneal cells, between the peritoneal cell and the lymphatic endothelial cell, and between the lymphatic endothelial cells. The lumen of the lymphatic capillary (L) is abundant in the carbon particles, whereas the peritoneal cavity $(P)$ is free from the carbon particles. The submesothelial connective tissue is shown at c. $\times 11,000$.

Fig. 9. A few carbon particles can be seen in the peritoneal cavity $(P)$ and in the tissue spaces beneath the peritoneal cell $(\mathrm{m})$. Abundant carbon particles are being transferred from the tissue spaces in the submesothelial connective tissue (c) through the two wide intercellular spaces between the lymphatic endothelial cells (e) to the lumen of a lymphatic capillary (L). $\times 9,000$.

Fig. 10. There are three intercellular spaces between the peritoneal cells (m), in which two spaces (arrow) are almost open from the peritoneal cavity (P) to the 
submesothelial tissue space (s) between the collagenous bundles (c), and the resting one is tightly closed. The peritoneal cells seems to take up the carbon particles from their free surfaces. Two vacuoles in the cytoplasm of the peritoneal cell are filled with the carbon particles. The carbon particles, moreover, can be seen in the submesothelial tissue space and in a dilation of the intercellular space between the two neighbouring endothelial cells (e) of a lymphatic capillary (L). $\times 14,500$.

\section{Plate 4}

Figs. 11 to 13 are from the animals killed 30 minutes after intraperitoneal injection of India ink.

Fig. 11. Abundant carbon particles can be seen passing from the peritoneal cavity $(P)$ through a wide intercellular space between the peritoneal cells (m) to a submesothelial space (s) between the collagenous bundles (c), covered at its major part by the cytoplasmic protrusions of the peritoneal cell and the lymyhatic endothelial cell. The vesicles in the cytoplasm of the peritoneal cells are particular richly found adjacent to the surface of three large invaginations of the plasma membrane. Numerous vesicles are also found in the cytoplasm of the lymphatic endothelial cell (e). The submesothelial tissue space communicates through an interrupted tubular space (arrow) between the lymphatic endothelial cells with the lumen of the lymphatic capillary (L). $\times 15,500$.

Fig. 12. Abundant carbon particles are being carried from the peritoneal cavity (P) through a canal to the lumen of a lymphatic capillary. The canal is first surrounded by the peritoneal cell $(\mathrm{m})$, secondly by the collagenous bundles (c), and finally by the lymphatic endothelial cells (e). $\times 20,500$.

Fig. 13. In the left one third of this figure, the peritoneal cell (m) linning over the submesothelial connective tissue $(c)$ is interrupted. The peritoneal cavity $(P)$ communicates through the interruption of the peritoneal covering and then through a wide space stuck by carbon particles between the collagenous bundles with the lumen of a lymphatic capillary (L). In the right one third, a lymphatic endothelial cell (e) is covered directly by the extremely thin cytoplasm of the two peritoneal cells. At the right side of this lymphatic endothelial cell the carbon particles lie in a wide communicating space between the peritoneal cavity and the lymphatic capillary. In the center one third, a few carbon particles can be seen in a narrow space beneath the peritoneal cell. This space seems to communicate with the lymphatic capillary at the opening (arrow), stuck by carbon particles, between the two lymphatic endothelial cells. $\times 14,000$.

Plate 5

Figs. 14 to 16 are from the animals killed 30 minutes after intraperitoneal injection of India ink.

Fig. 14. A few carbon particles lie in the peritoneal cavity $(P)$ at the base of the microvilli-like processes of the peritoneal cells $(\mathrm{m})$. In the peritoneal layer there are two tubular intercellular spaces (arrow), opening to the peritoneal cavity. A large, local dilation of the left intercellular space is filled with abundant carbon particles. A few carbon particles can be seen in the small vacuoles in the cytoplasm of the peritoneal cell. The carbon particles are also found in the tissue spaces beneath the peritoneal cells. Beyond the submesothelial connective tissue (c), the carbon particles can be seen abundantly in the tissue spaces beneath the 
lymphatic endothelial cells (e). A few carbon particles are in the lumen of a lymphatic capillary (L). $\times 18,500$.

Fig. 15. This photograph shows that a large dilation of the intercellular space between the peritoneal cells in figure 14 is connected with the lumen of the lymphatic capillary (L) through a space between the submesothelial collagenous bundles (c) and then through a space between the lymphatic endothelial cells (e). The carbon particles stick to these spaces. A few carbon particles in the peritoneal cavity $(P)$ are at the base of the microvilli-like processes. $\times 19,500$.

Fig. 16. The peritoneal cavity (P) communicates through a canal-like intercellular space (arrow) between the two peritoneal cells (m) with a large submesothelial space between the collagenous bundles (c). The submesothelial space is separated by a thin, interrupted lymphatic endothelial cell (e) layer from the lumen of a lymphatic capillary (L). The carbon particles are a few in number in the peritoneal cavity and in the near portion of the intercellular space to the submesothelial space. Abundant carbon particles can be seen in the large submesothelial space. The carbon particles, moreover, can be found in a few small vacuoles and in a large vacuole in the cytoplasm of the peritoneal cell. $\times 24,500$.

Plate 6

Figs. 17 and 18 are from the animals killed 60 minutes after intraperitoneal injection of India ink.

Fig. 17. The peritoneal cavity $(P)$ is empty in the left upper corner. The free surface of the peritoneal cells $(m)$ is attached by the extremely small carbon particles. A intercellular space between the two peritoneal cells is tightly closed. The carbon particles are included in various shape and sized vacuoles in the cytoplasm of the left peritoneal cell. A vacuole (arrow) is apparently discharging the carbon particles into a submesothelial tissue space (s). The surface of the submesothelial collagenous bundles (c) is almost wholly covered by the thin cytoplasm of the lymphatic endothelial cells (e). The cytoplasmic protrusion of a lymphatic endothelial cell, covering the left collagenous bundle, is in direct contact with the peritoneal cell. The carbon particles can be seen passing from the submesothelial space (s) through an intercellular space between the lymphatic endothelial cells into the lumen of a lymphatic capillary (L). $\times 14,000$.

Fig. 18. The free surface of the peritoneal cells (m) is attached by the extremely small carbon particles. In the left upper corner, the peritoneal cavity (P) opens through a space (arrow) between the two widely separated peritoneal cells to a large submesothelial space (s). The submesothelial space is separated by a lymphatic endothelial cell (e) layer from the lumen of a lymphatic capillary (L). A thin cytoplasmic process of a lymphatic endothelial cell streches on the surface of the collagenous bundle (c) and contacts directly with the peritoneal cell. The carbon particles can be seen in two vacuoles and in a opaque body (o) in the cytoylasm of the peritoneal cell. Various shape and sized vacuoles in the cytoplasm of the lymphatic endothelial cells are filled with abundant carbon particles. Moreover, a few carbon particles are contained in a large dilation (arrow) of the intercellular space between the lymphatic endothelial cells. $\times 16,000$.

Plate 7

Figs. 19 and 20 are from the animals killed 60 minutes after intraperitoneal injection of India ink. Fig. 21 is from an animals killed 30 minutes and Fig. 22 is from an animal killed 15 minutes after injection. 
Fig. 19. The lymphatic endothelial cell (e) layer is apparently exposed to the peritoneal cavity $(P)$ as the peritoneal cavity communicates through a wide opening (arrow) between the two separated peritoneal cells (m) with a large submesothelial space (s). The surface of the thin cytoplasm of the lymphatic endothelial cell is attached on the peritoneal cavity side by the extremely small carbon particles, as well as the surface of the peritoneal cells. Two large vacuoles in the cytoplasm of the lymphatic endothelial cell are filled with abundant carbon particles. A small vacuole, situated a little lower in this figure than the large vacuoles, is apparently discharging the carbon particles into the lumen of a lymphatic capillary. A portion of the submesothelial collagenous bundle is shown at c. $\times 21,000$.

Fig. 20. The free surface of the peritoneal cell (m) is heavily loaded with the extremely small carbon particles. In the cytoplasm of the peritoneal cell, the carbon particles can be seen in two small vacuoles and in a opaque body (o). The opaque body contains a uncertain oval structure with the carbon granules. A large space (s) in the submesothelial connective tissue (c) includes a large number of the carbon particles, among which a few carbon particles lie at the base of the small microvilli-like processes (arrow) of the lymphatic endothelial cell (e). Moreover, the carbon particles seem to stick to the surface of the lymphatic endothelial cell. In the cytoplasm of the lymphatic endothelial cell, the carbon particles can be seen in the small vacuoles. The lumen of the lymphatic capillaries is indicated at $\mathrm{L} . \times 14,000$.

Fig. 21. A peritoneal cell (m) is apparently taking up the carbon particles from the peritoneal cavity (P) and discharging them (arrow) from the phagocytotic vacuoles into the large submesothelial space (s). A opaque body (o) in the cytoplasm of the peritoneal cell includes a few very small carbon granules with a oval uncertain structure. The submesothelial space, separated by the thin cytoplasm of the lymphatic endothelial cell (e) from the lumen of a lymphatic capillary $(L)$, is divided into three spaces by transversely or obliquely running cytoplasmic processes of the lymphatic endothelial cell. Each spaces communicate with each other through the interruption space between the processes. $\times 22,000$.

Fig. 22. This photograph shows a portion of a peritoneal cell. A few carbon particles situate in the peritoneal cavity $(P)$ at the base of the microvilli-like processes of the peritoneal cell. A opaque body (o) includes a number of extremely small carbon granules with a few large particles and a oval uncertain structure. $\times 30,000$. 
Plate I
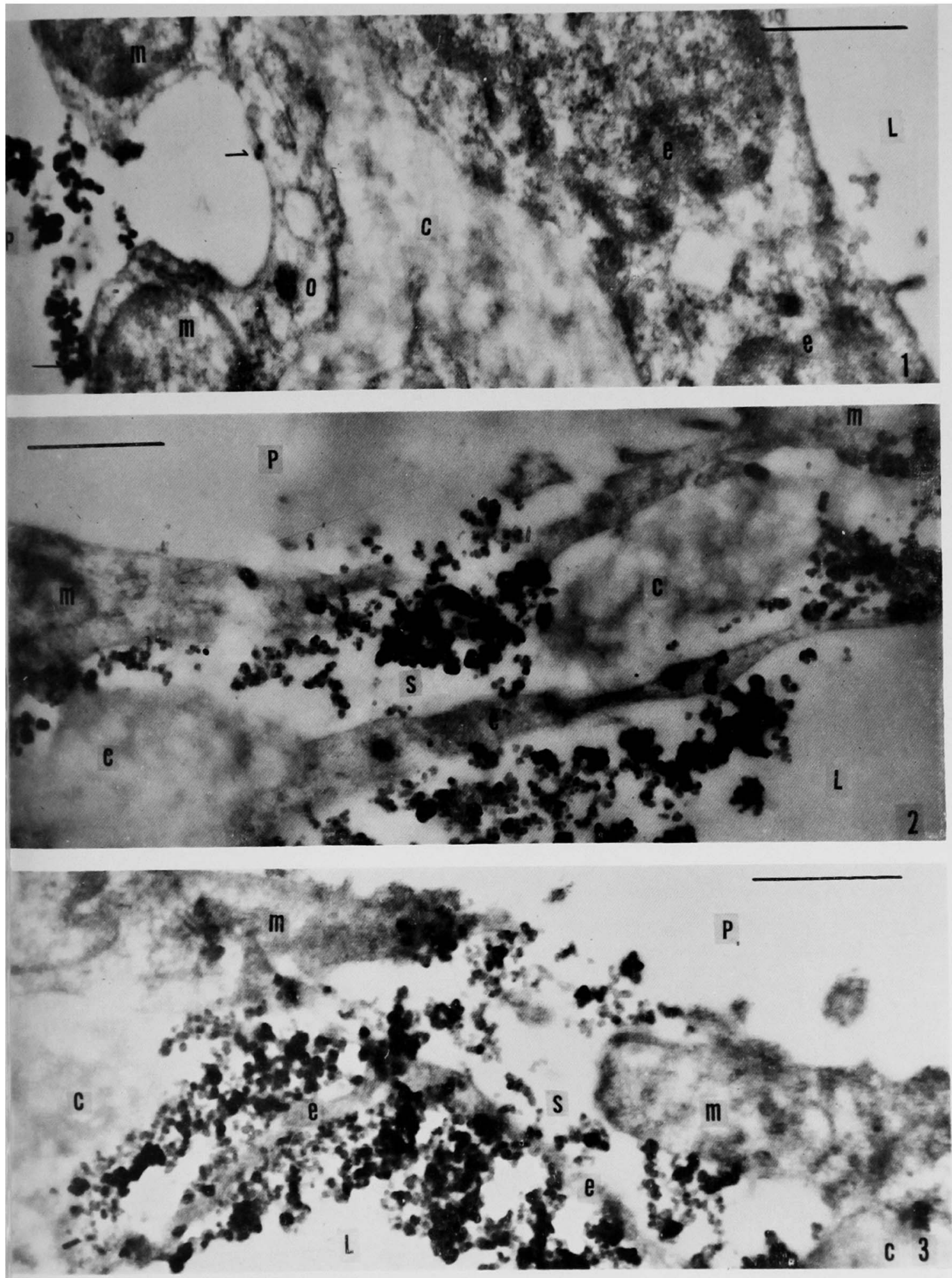
Plate II
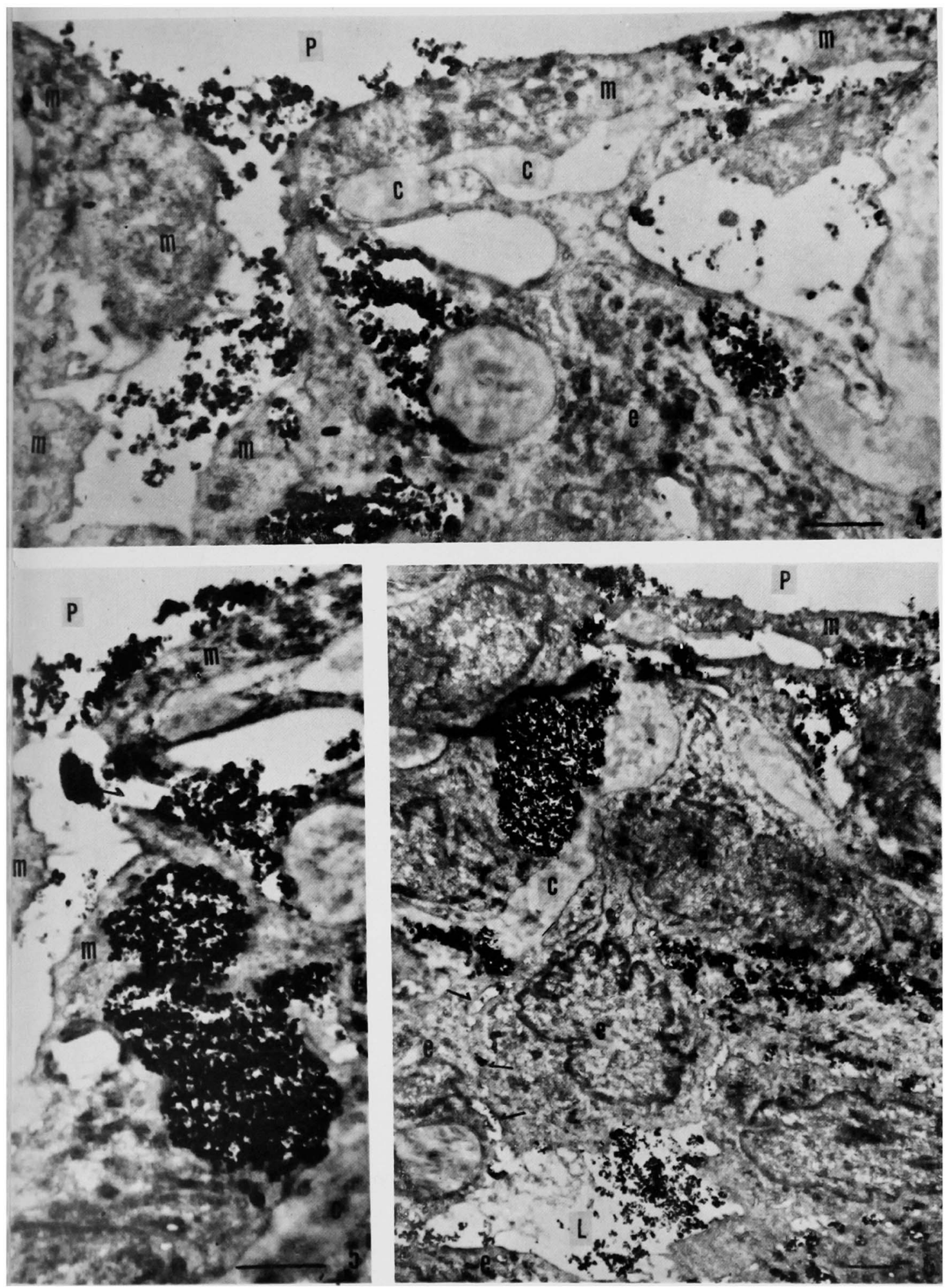

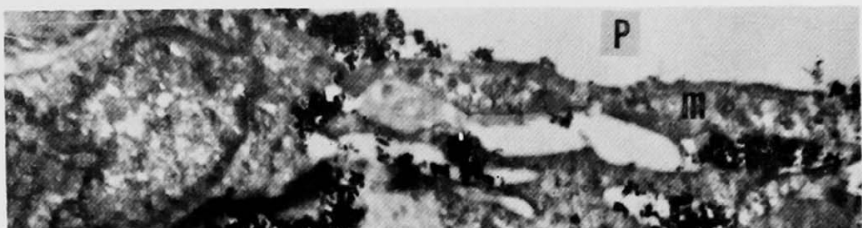
(6)

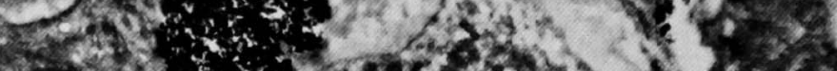

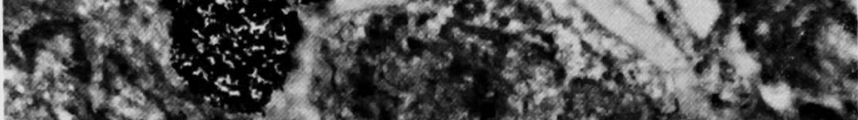

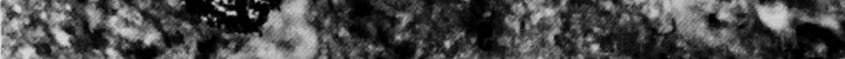

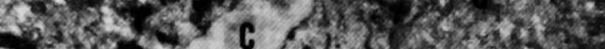

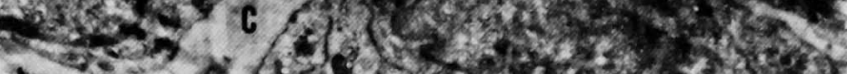

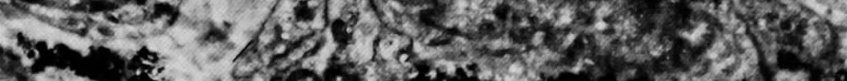

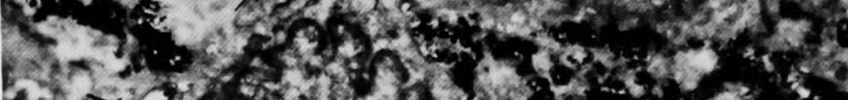

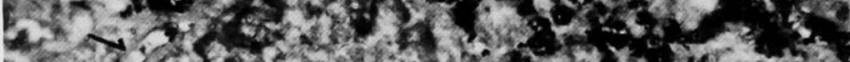

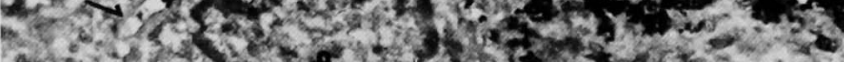

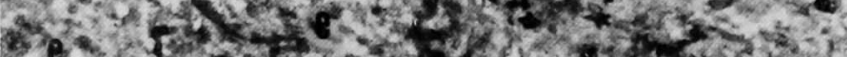

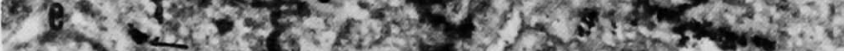

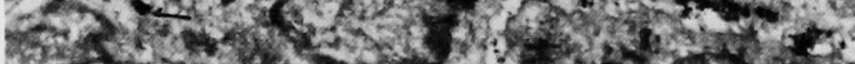

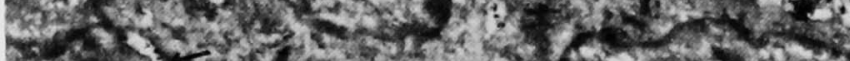

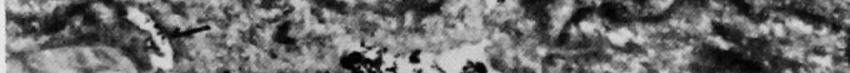

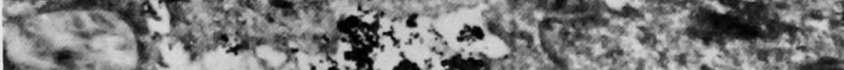

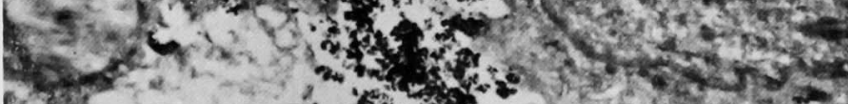

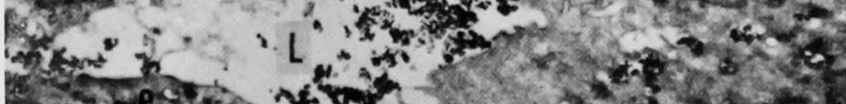

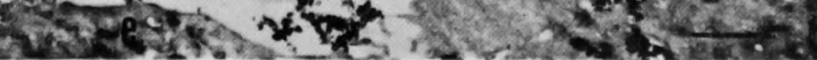


Plate III
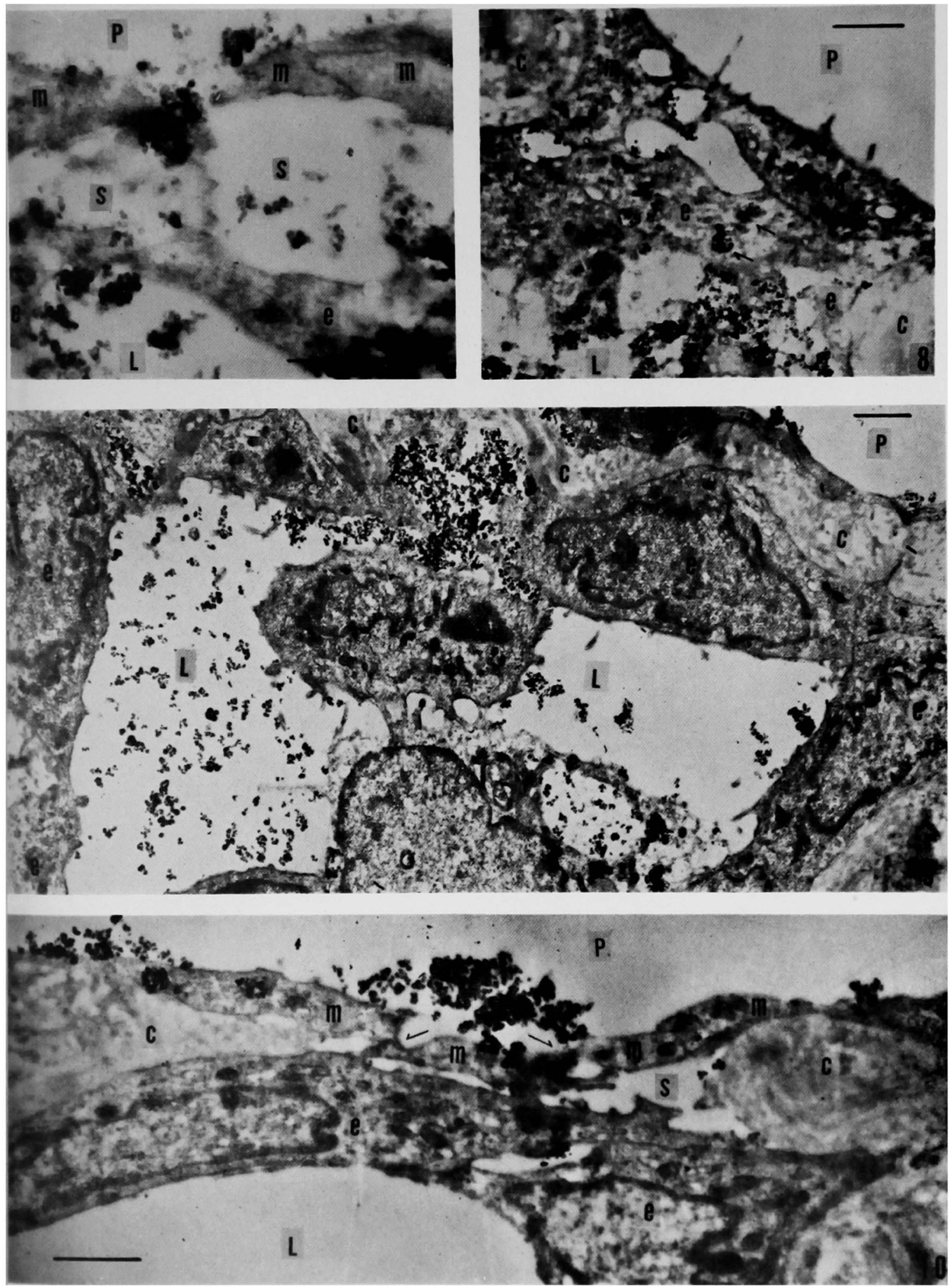

M. Kotani, M. Rai and S. Nakano 

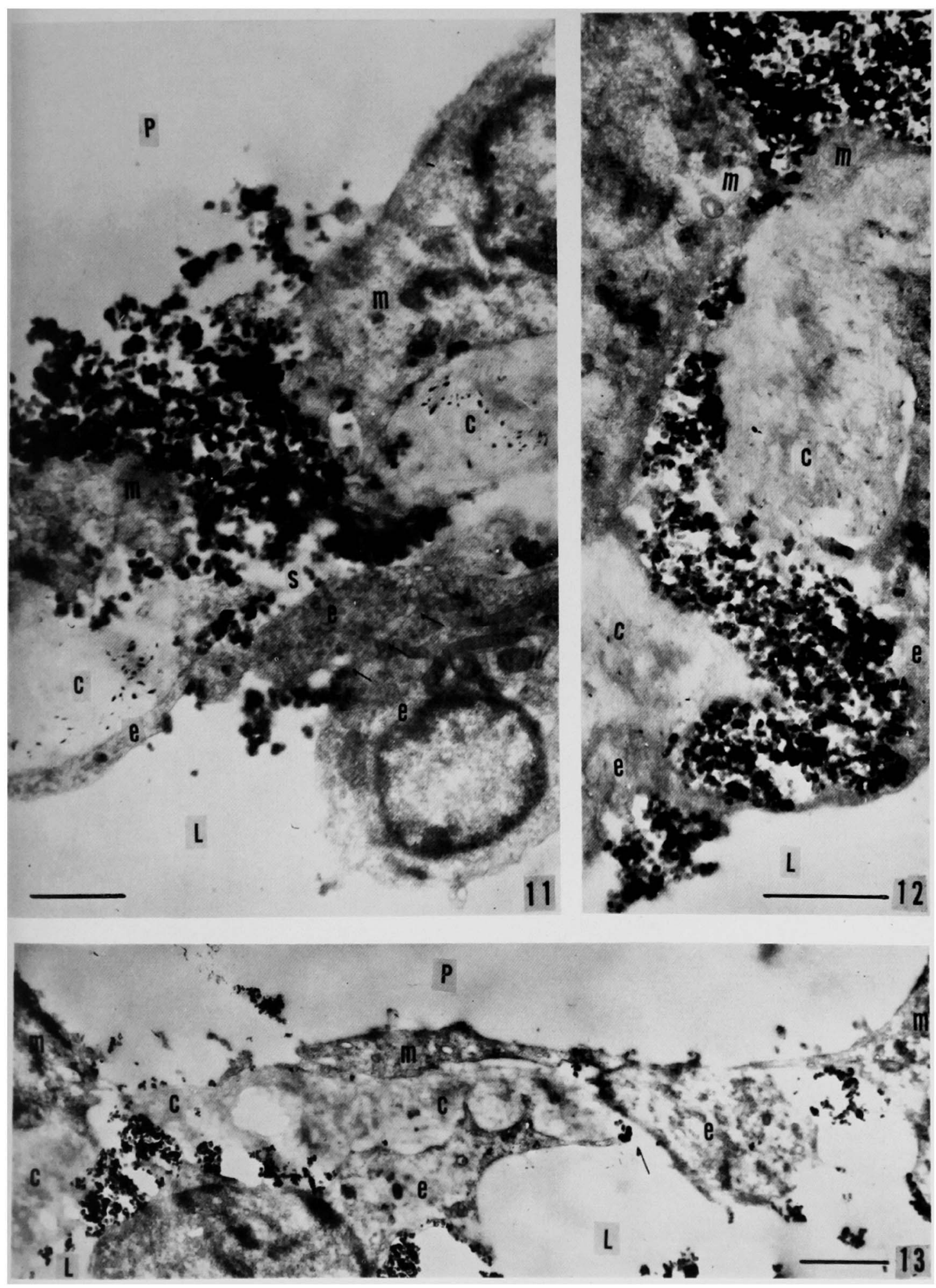
Plate V
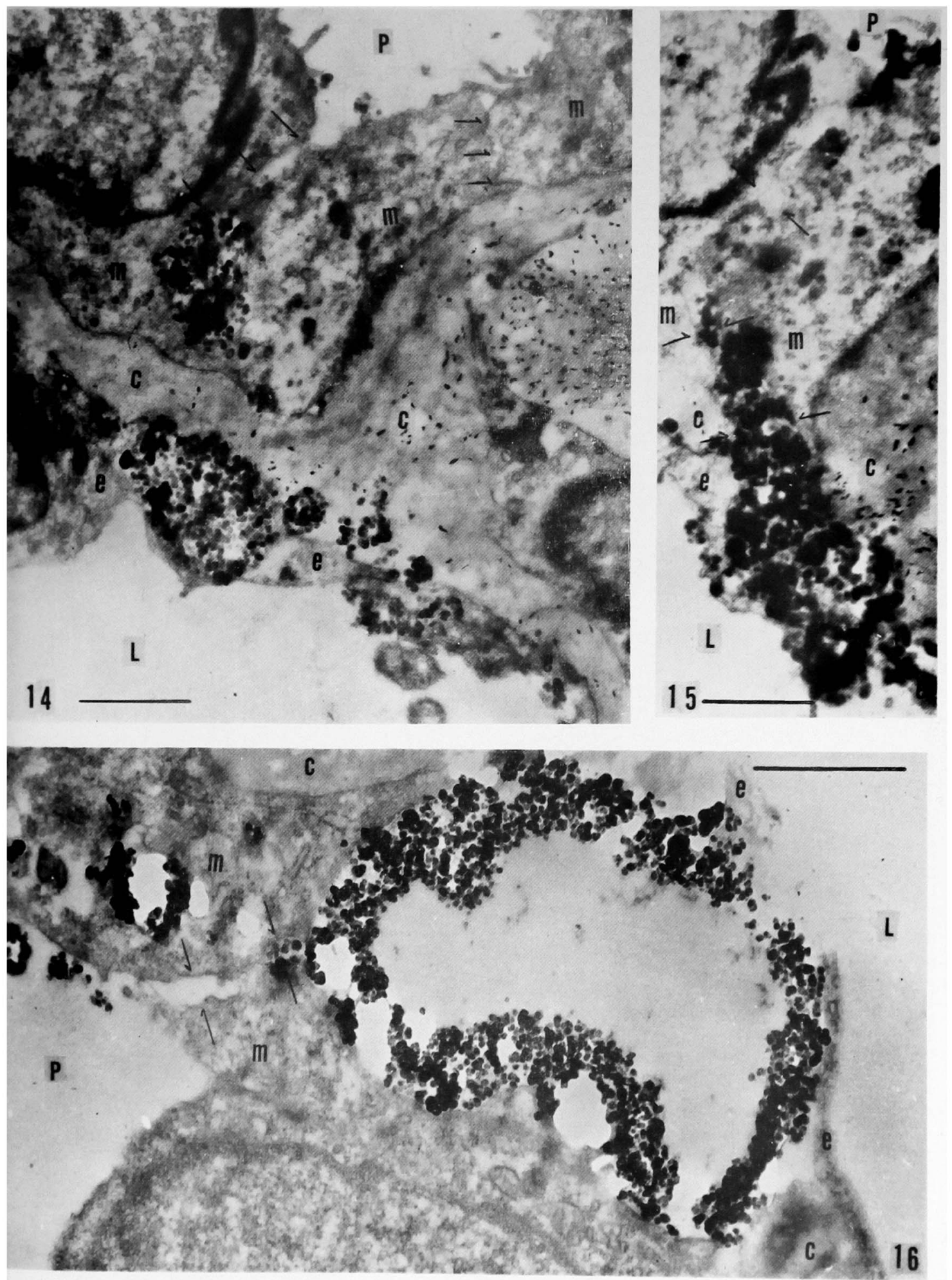
Plate VI
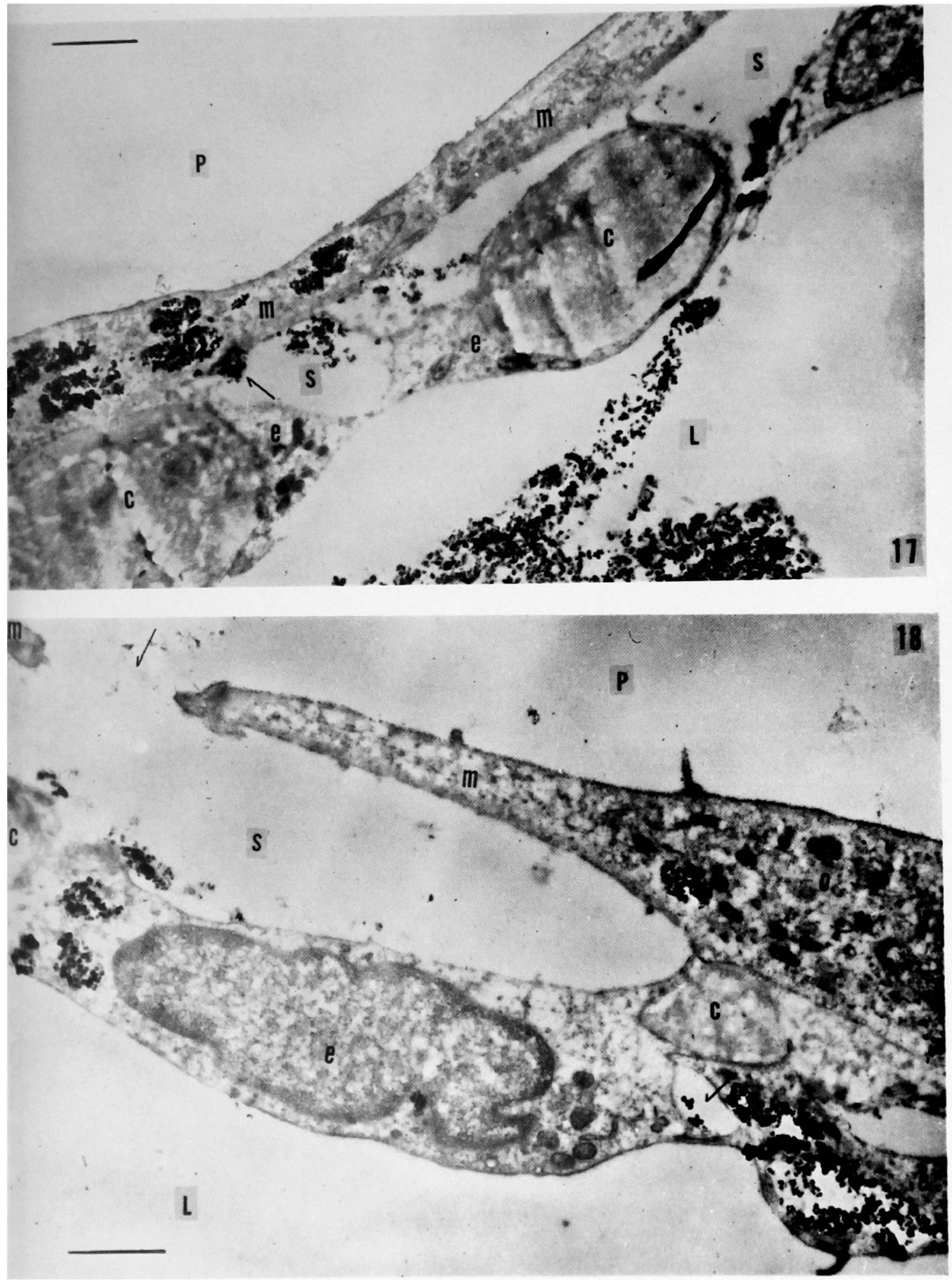

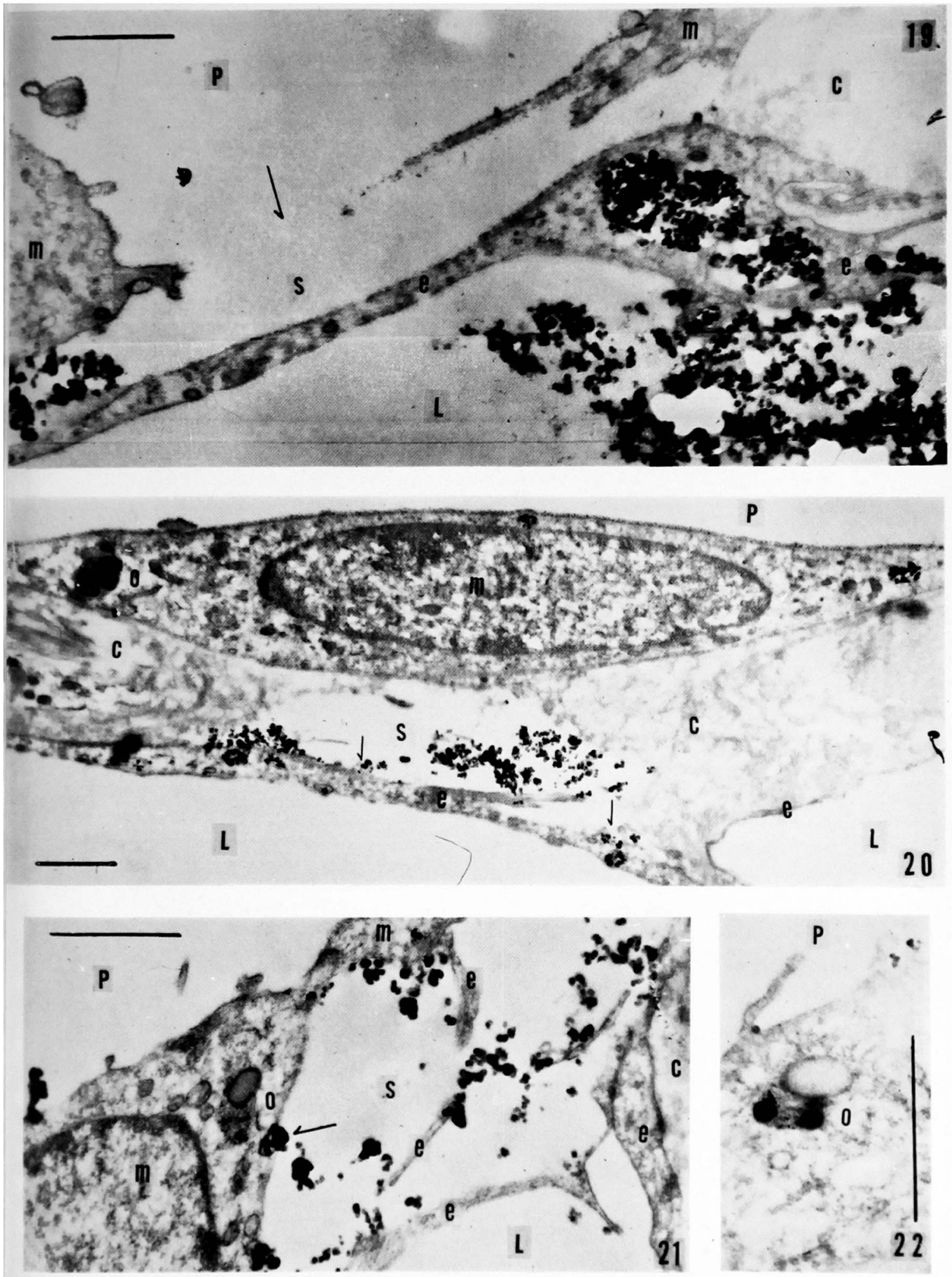

M. Kotani, M. Rai and S. Nakano 